\title{
Fractionation of Poly(ethylene glycol) by High Osmotic Pressure Chromatography
}

\author{
Shigetomo MatsuYama, ${ }^{\dagger}$ Hisae NaKaHARA, Kazuhiko TaKeUChI, \\ Ritsuko Nagahata, Shinichi Kinugasa, and Iwao Teraoka* \\ National Institute of Materials and Chemical Research, \\ 1-1 Higashi, Tsukuba, Ibaraki 305-8565, Japan \\ *Department of Chemical Engineering, Chemistry, and Materials Science, Polytechnic University, \\ 333 Jay Street, Brooklyn, New York 11201, U.S.A.
}

(Received August 13, 1999)

\begin{abstract}
Poly(ethylene glycol) (PEG) was fractionated by high osmotic pressure chromatography (HOPC) with water and 1,4-dioxane as eluents. Using dimethyloctylchlorosilane $\left(\mathrm{C}_{8}\right)$-modified controlled-pore glass (CPG) with 1,4dioxane as eluent, high molar mass (MM) PEG components were fractionated. The average MM of the fractionated PEG samples strongly depends on the eluent and the polarity of column packing materials. PEGs dissolved in water were adsorbed onto the column packing materials more easily compared with 1,4-dioxane. Comparison between simulation and experiments indicated that adsorption of PEG molecules onto the surface of the column packing materials reduces the separation efficiency of HOPC fractionation. By preventing adsorption, PEG components with higher MM and narrow molar mass distribution (MMD) were fractionated even from a commercial PEG with narrow MMD.

KEY WORDS High Osmotic Pressure Chromatography / Poly(ethylene glycol) / Fractionation / Molar Mass / Molar Mass Distribution / Adsorption /
\end{abstract}

Polymer samples with narrow molar mass distribution (MMD) are frequently used as reference materials for size exclusion chromatography (SEC). These polymers are commonly synthesized by living polymerization, but often contain monomers, oligomers, and dimerization products whose average molar mass (MM) is twice as large as the peak MM.

There are several separation methods to prepare polymers with narrow MMDs from polymers with broad MMDs. Preparative SEC and fractional precipitation ${ }^{1}$ are widely used to separate polymers. These methods consume a large amount of solvent, and thus high osmotic pressure chromatography (HOPC) was recently proposed by Teraoka et al. ${ }^{2-4}$ HOPC consumes a much smaller volume of solvent compared the other methods. Therefore HOPC is expected to be an excellent method for environment.

In studies of HOPC hitherto reported, hydrophobic polymers such as polystyrene were fractionated in organic solvents in various experimental conditions. ${ }^{3-5}$ In contrast, only a few experimental studies have been carried out for water-soluble polymers. ${ }^{6}$ Because poly(ethylene glycol) (PEG) is a typical water-soluble polymer often used as a reference material for aqueous SEC, we studied the efficiency of HOPC separation for PEG using water and 1,4-dioxane as eluents. We investigated changes in the average MM and the MMD of PEG fractions separated during HOPC. We used HOPC to further narrow the MMD of commercial PEG samples with already narrow $\mathrm{MMD}$.

\section{HIGH OSMOTIC PRESSURE CHROMATOGRAPHY}

HOPC uses columns packed with solvent-imbibed porous materials. Figure 1 shows a schematic view of HOPC. Unlike Teraoka's system, ${ }^{2}$ a sample loop con-

\footnotetext{
${ }^{\dagger}$ To whom correspondence should be addressed.
}

nected to a switching valve (Rheodyne 7000) was used to store a viscous solution of polymer with a concentration higher than the overlap concentration $c^{*}$. In this study, $c^{*}$ is defined as $c^{*}\left(2^{1 / 2} R_{\mathrm{G}, 0}\right)^{3}=M / N_{\mathrm{A}}{ }^{7}$ where $R_{\mathrm{G}, 0}$ is the radius of gyration of the polymer chain in the dilute solution limit, $M$ the molar mass, and $N_{\mathrm{A}}$ Avogadro's number. The volume of the sample loop was selected to be close to that of the mobile phase in the HOPC column. The solution was slowly injected into the column by switching the valve. The eluent was collected into test tubes until it became a pure solvent. HOPC was then ready for the next batch of processing.

The separation mechanism of HOPC can be explained on the basis of size exclusion and repulsion between polymer molecules at high concentrations. When a porous medium is equilibrated with concentrated polymer solution, the high osmotic pressure of the mobile phase (exterior to the porous materials) drives polymer molecules into the stationary phase (interior of the porous materials). ${ }^{8}$ The ratio of the polymer concentration in the pore to that in the surrounding solution is called the partition coefficient $K$. When there is no MMD for the polymer, $K$ never exceeds unity, although $K$ approaches unity with increasing concentration of polymer. The partitioning is different, however, when the solute is a polymer with a broad MMD. When polymer chains are forced into the confined geometry, loss of the conformation entropy for lower MM chains is smaller compared with higher MM chains. For low MM components, $K$ easily exceeds unity, whereas $K$ for high MM components is smaller than in the absence of low MM components. ${ }^{9}$ Forced migration favors low MM components, ${ }^{9}$ thereby enriching the mobile phase with high MM components and the stationary phase with low MM components (enhanced partitioning). ${ }^{10}$

In HOPC, enhanced partitioning between the mobile phase and stationary phase is repeated at each plate as the mobile phase is transported to the next plate. The 


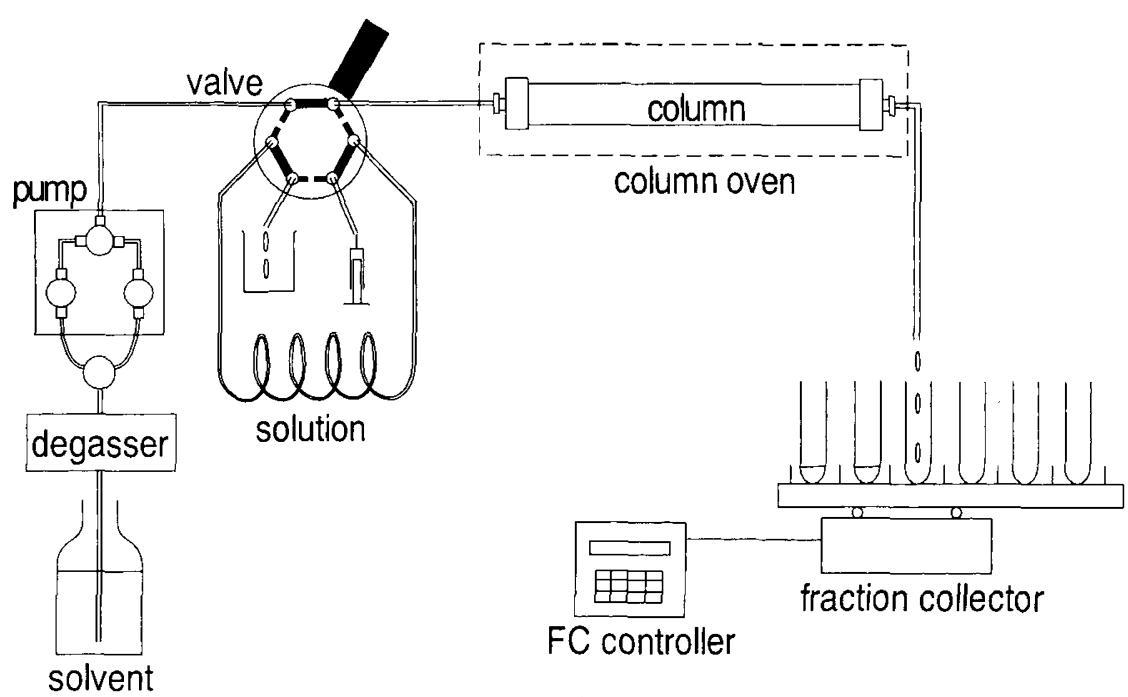

Figure 1. Schematic of a high osmotic pressure chromatography system.

Table I. Characteristics of Poly(ethylene glycol) Samples

\begin{tabular}{ccccccc}
\hline Sample & $M_{p}{ }^{\mathrm{a}}$ & $M_{w}{ }^{\mathrm{b}}$ & $M_{w} / M_{n}{ }^{\mathrm{b}}$ & $R_{\mathrm{G}, \mathrm{aq}} / \mathrm{nm}^{\mathrm{c}}$ & $R_{\mathrm{G}, \theta} / \mathrm{nm}^{\mathrm{c}}$ & $c^{*} / \mathrm{g} \mathrm{cm}^{-3 \mathrm{~d}}$ \\
\hline PEG 2K & 1900 & 1900 & 1.02 & 1.61 & 1.38 & 0.27 \\
PEG 3K & 2900 & 2900 & 1.02 & 2.05 & 1.69 & 0.20 \\
\hline${ }^{\mathrm{a}}$ Observed peak molar mass. ${ }^{\mathrm{b}}$ Calculated from SEC chromatograms. ${ }^{\mathrm{c}}$ Estimated from ref 9 and 10. & ${ }^{\mathrm{d}}$ In water.
\end{tabular}

front end of the transported solution is equilibrated each time with a pure solvent in the stationary phase, thereby eliminating lower MM components from the mobile phase. When the front end reaches the column outlet, it is enriched with the highest MM components originally present in the injected solution. The next portion of the mobile phase that follows the front end exchanges the polymer chains between the mobile phase and stationary phase that already has low MM components. Removal of these components from the mobile phase is not as easy as for the front end. Thus, the average MM decreases and distribution broadens as the number of fractions increases.

\section{EXPERIMENTAL}

\section{Materials}

Two commercial PEG samples purchased from Wako Pure Chemical Industries Ltd. were used without further purification. Table I shows their characteristics. The peak MM, $M_{p}$, mass-average MM, $M_{w}$, and polydispersity index $M_{w} / M_{n}$ were calculated from the SEC chromatograms without correction for band broadening, where $M_{n}$ is the number-average MM. The radius of gyration in the dilute aqueous solution limit, $R_{\mathrm{G}, \mathrm{aq}} \equiv$ $\left\langle S_{\mathrm{aq}}^{2}\right\rangle^{1 / 2}$, and that in a theta solvent, $R_{\mathrm{G}, \theta}$, calculated by dividing the end-to-end distances by $6^{1 / 2}$, are from the literature. ${ }^{11,12}$

Controlled-pore glass (CPG; CPG-10, 200/400 mesh) was purchased from CPG Inc. and used as column packing material. According to the manufacturer, the mean pore diameter of the CPG is $8.5 \mathrm{~nm}$, pore volume, 0.37 $\mathrm{cm}^{3} \mathrm{~g}^{-1}$, and surface area, $224.5 \mathrm{~m}^{2} \mathrm{~g}^{-1}$

Before use, the CPG beads were washed with $50 \%$ ni-
Table II. Separation Conditions of HOPC

\begin{tabular}{|c|c|c|c|c|}
\hline \multirow[b]{2}{*}{ Experiments } & \multicolumn{2}{|c|}{ Weight fraction } & \multirow{3}{*}{ Solvent } & \multirow{3}{*}{ Type of CPG } \\
\hline & PEG2K & PEG3K & & \\
\hline & $\mathrm{wt} / \mathrm{wt} \%$ & $\mathrm{wt} / \mathrm{wt} \%$ & & \\
\hline Exp-A & 35.0 & & 1,4-Dioxane & CPG-C ${ }_{8}{ }^{a}$ \\
\hline$-B$ & 35.0 & & $\mathrm{H}_{2} \mathrm{O}$ & $\mathrm{CPG}-\mathrm{C}_{8}$ \\
\hline$-\mathrm{C}$ & 30.0 & & $\mathrm{H}_{2} \mathrm{O}$ & $\mathrm{CPG}-\mathrm{OH}{ }^{\mathrm{b}}$ \\
\hline$-D$ & 35.0 & & $\mathrm{H}_{2} \mathrm{O}$ & CPG-OH \\
\hline$-E$ & 35.0 & & 1,4-Dioxane & CPG-OH \\
\hline$-F$ & 70.0 & & 1,4-Dioxane & $\mathrm{CPG}-\mathrm{C}_{8}$ \\
\hline$-G$ & 70.0 & & $\mathrm{H}_{2} \mathrm{O}$ & $\mathrm{CPG}-\mathrm{OH}$ \\
\hline$-\mathrm{H}$ & - & & 1,4-Dioxane & CPG-C ${ }_{8}$ \\
\hline$-I$ & - & & $\mathrm{H}_{2} \mathrm{O}$ & CPG-OH \\
\hline
\end{tabular}

${ }^{a} \mathrm{CPG}$ chemically modified with dimethyloctyl $\left(-\mathrm{C}_{8}\right) \cdot{ }^{\mathrm{b}} \mathrm{CPG}$ washed by $\mathrm{HNO}_{3}$.

tric acid at $70-80^{\circ} \mathrm{C}$ overnight to remove organic impurities and rinsed thoroughly with water until the supernatant liquid became neutral. The cleaned CPG beads were dried in a convection oven, and divided into two portions : one used as it was and the other chemically modified with dimethyloctylchlorosilane $\left(\mathrm{C}_{8}\right)^{4,13}$ The surface of the former CPG is chemically covered with hydroxy group (-OH) and designated as CPG-OH in the present paper. The latter CPG covered with dimethyloctyl group $\left(-\mathrm{C}_{8}\right)$ is designated as CPG-C $8 .{ }^{14}$ The surface polarity of $\mathrm{CPG}-\mathrm{OH}$ is greater than that of $\mathrm{CPG}-\mathrm{C}_{8}$.

Table II shows conditions of nine HOPC experiments (Exp-A through Exp-I) conducted. In Table II, the weight fractions of PEGs in solutions injected are shown together with solvents and CPGs used. Solid samples of PEG2K and PEG3K were mixed first and then dissolved in 1,4-dioxane (Exp-A and -E) and water (Exp-B, -C, and -D). PEG2K and PEG3K were also dissolved in 1,4dioxane (Exp-F and -H) and water (Exp-G and -I) sepa- 
7

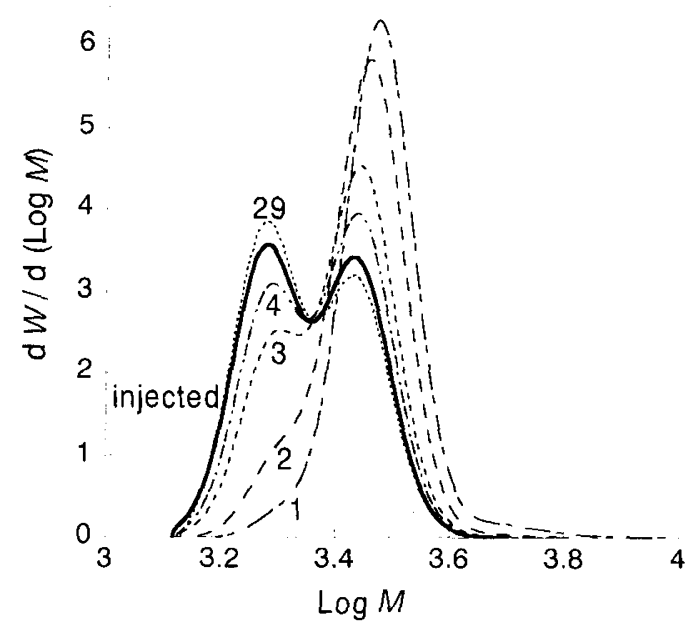

Figure 2. Differential molar mass distribution $\mathrm{d} W / \mathrm{d} \log M$ as a function of $\log M$ for fractions separated from a PEG2K/PEG3K mixture. HOPC separation was carried out with 1,4-dioxane as an eluent and CPG- $\mathrm{C}_{8}$ as stationary phase. The number adjacent to each line represents the fraction number.

rately. In Exp-A, -B, -F, and - $\mathrm{H}, \mathrm{CPG}-\mathrm{C}_{8}$ was used as column packing material. In Exp-C, -D, -E, -G, and -I, CPG$\mathrm{OH}$ was used.

Ion-exchanged water was used in the present study. 1,4-Dioxane (Wako Pure Chemical Industries Ltd.) was used without purification.

\section{Fractionation by HOPC}

Before injection, the column was washed with the same solvent as used to prepare the solution. For Exp-B, 1,4-dioxane filling the $\mathrm{CPG}-\mathrm{C}_{8}$ column was replaced first by tetrahydrofuran, then by methanol, and finally by water to ensure that the most of the solvent in the stationary phase is water. The columns used in HOPC were made of stainless steel and had dimensions of $4.0 \mathrm{~mm}$ i.d. $\times 30 \mathrm{~cm}$ length. PEG solutions filled in a sample loop $(2$ $\mathrm{cm}^{3}$ ) were injected into the column at $40^{\circ} \mathrm{C}$ at the flow rate of $0.1 \mathrm{~cm}^{3} \mathrm{~min}^{-1}$. Early fractions were collected 10 drops each, and later fractions 50-250 drops each. The column was fully washed with solvent until the eluent became pure solvent. The solvent was evaporated in all fractions by blowing hot nitrogen gas. After the evaporation, the collected PEG fractions were weighed by a balance.

\section{Characterization of Polymers}

SEC was used to determine average MMs and polydispersity index $M_{w} / M_{n}$ of the PEG samples. A Tosoh SEC system coupled with a Model DP-8020 pump and a Model RI-8020 differential refractometer was used. Two SEC columns (Shodex OHpak SB-802.5 HQ) were operated at $40^{\circ} \mathrm{C}$. The mobile phase was water, and the flow rate was $1.0 \mathrm{~cm}^{3} \mathrm{~min}^{-1}$. The concentration of the polymer solution was low to avoid MM dependence of the chromatogram, which was typically between 0.10 and $0.02 \mathrm{wt} \%$, depending on MM of the analyte. Each set of columns was calibrated with PEG reference materials of MM from 194 to 22800 purchased from Polymer Laboratories.

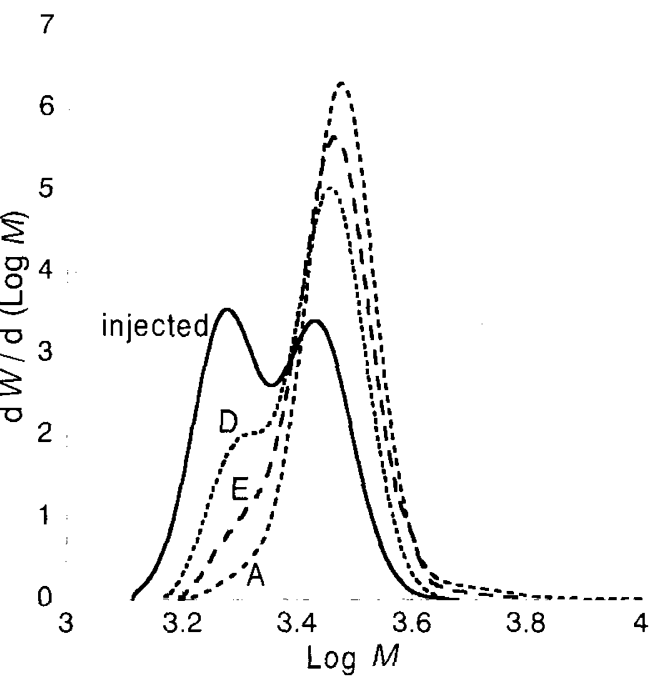

Figure 3. Comparison of the molar mass distribution for the first fractions in (A) Exp-A, (D) Exp-D, (E) Exp-E, and the PEG mixture injected. For experimental conditions, see Table II.

The reflective type matrix-assisted laser desorption/ ionization time-of-flight mass spectrometry (MALDITOFMS ; Shimadzu/KRATOS KOMPACT MALDI III) equipped with a $337 \mathrm{~nm}$ nitrogen laser was also used to determine average MMs and MMDs of the PEG samples. The matrix used in the MALDI-TOFMS measurements was 2,5-dihydroxybenzoic acid.

\section{RESULTS AND DISCUSSION}

\section{Separation of Mixed PEGs}

Figure 2 shows examples of differential MMD for some of the PEG fractions (dashed and dotted lines) collected in Exp-A. MMD of the original mixed PEG sample injected is shown by a thick solid line. All differential MMDs in Figure 2 were calculated from SEC chromatograms. Numbers in Figure 2 represent fraction numbers. Fraction 1 has the highest MM and the narrowest MMD. $M_{p}$ of the fraction 1 is slightly larger than that of original PEG3K before mixing, and $M_{w} / M_{n}$ of fraction 1 is close to that of the original PEG3K. The ratio of PEG$3 \mathrm{~K}$ component to PEG2K component in each fraction decreases as the fraction number increases. Finally, MMDs of the late fractions were close to that of the injected PEG mixture. The tendency of changing MMD in the fractions collected agrees well with previous HOPC fractionation results. ${ }^{2-5}$

The separation was not successful in Exp-B, where water was used as an eluent in place of 1,4-dioxane. The same $\mathrm{CPG}-\mathrm{C}_{8}$ was used as packing material. In Exp-B, MMDs of all eluted fractions were not different from the MMD of the injected PEG sample. The difference in polarity between water and 1,4-dioxane may have caused the difference. We speculate that the polar solvent hardly permeated the pore covered with a nonpolar material, $\mathrm{C}_{8}$. PEG dissolved in water could not penetrate the pore.

Exp-C and Exp-D differ only in the concentration of PEG sample injected. The behavior of the eluent in these experiments was similar (not shown). MMs were slightly 


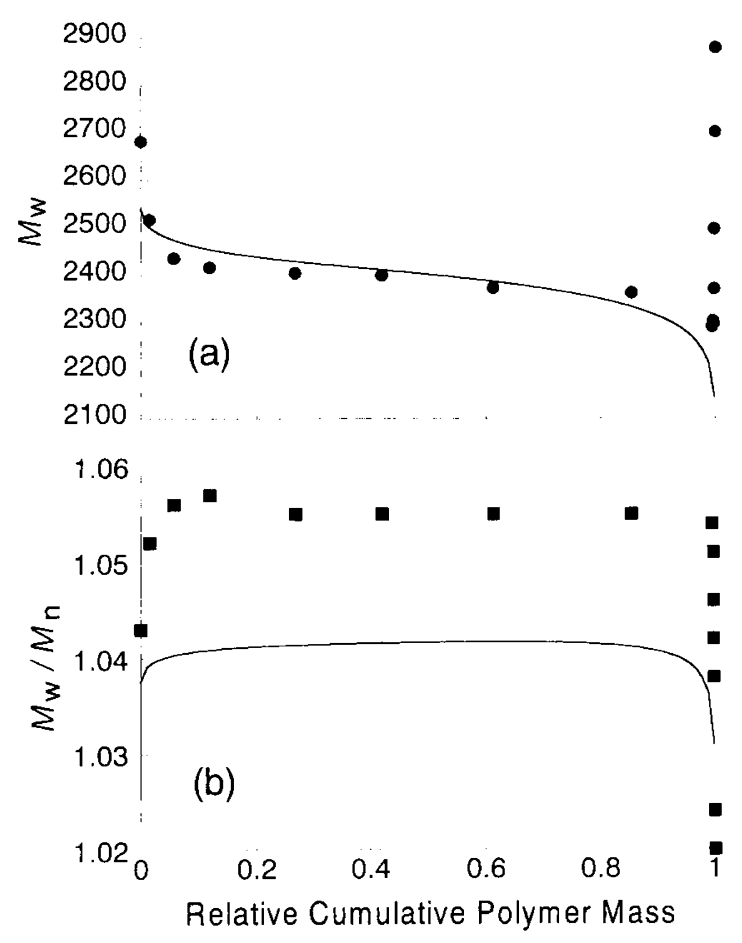

Figure 4. Comparison between simulation (solid line) and experiment (points) for Exp-D : (a) $M_{w}$ and (b) $M_{w} / M_{n}$ are plotted as a function of relative cumulative mass of PEG fractions, defined as a ratio of cumulative mass of eluted PEG to the mass of injected PEG.

higher and MMDs were narrower for each fraction in Exp-D compared with Exp-C. According to Teraoka et $a l .{ }^{9}$ the higher the polymer concentration, the larger the $K$ is for lower MM components. Therefore, the polymer solution with higher concentration produces a fraction with higher MM and narrower MMD. The results of Exp-C and Exp-D agreed well with theory.

Figure 3 compares differential MMD of the first PEG fraction in Exp-A, -D, and -E. The narrowest MMD for fractionated PEG samples was observed in Exp-A, and the broadest in Exp-D. The difference between Exp-D and Exp-E was the eluent and solvent used to dissolve PEG. A polymer with higher MM and narrower MMD was obtained in early fractions on using good solvents as eluent rather than poor solvents. ${ }^{5,15}$ For PEG, it could be expected that using water will produce fractions with higher MM and narrower MMD because it is a better solvent for PEG than 1,4-dioxane is. ${ }^{16}$ However, our experiments gave the opposite result. A possible explanation is adsorption of PEG molecules onto the surface of CPG-OH in Exp-D. Adsorption should have changed MMs and MMDs of fractionated PEGs. ${ }^{9}$ Figure 3 shows that even Exp-E produced a broader MMD with smaller $M_{w}$ for the first fraction than Exp-A did. This difference can also be ascribed to adsorption. The polar silanol surface could adsorb PEG molecules more easily than the nonpolar surface can in Exp-A.

\section{Simulation for HOPC Separation}

Partitioning of solvated polymer molecules with a bimodal MMD between a porous medium and an external mobile phase was investigated by Teraoka et al. ${ }^{9}$ using the results obtained in renormalization group theory. ${ }^{17}$
They only discussed partitioning at one plate. To understand separation through a column in HOPC, we conducted simulation of HOPC for separation by applying the theory of Teraoka et al.

The osmotic pressure of the semidilute solution and conformational entropy of the polymer chains need to be known to calculate HOPC for separation. For calculation of the osmotic pressure, we used a modified Ohta-Oono equation. ${ }^{17,18}$ The conformational entropy of the polymer chain in a pore is evaluated from $R_{\mathrm{G}, 0}$ and pore radius. We estimated $R_{\mathrm{G}, \text { aq }}$ of PEG in water from the results of Kawaguchi et al. ${ }^{11}$ Since the radius of gyration of PEG in dioxane is not known, we compare the separation with water only.

In HOPC, enhanced partitioning between the exterior and interior of porous materials is continuously repeated in each plate. Since the number of plates in the column cannot be known, we assumed two cases in which the column had 5 and 10 plates. There was little difference in the separation characteristics between 5 and 10 plates. We therefore discuss separation behavior for 5 plates only.

Our simulation assumed that the two polymers have no MMD before mixing. The observed peak-average MMs, $M_{p}$, of PEG were used as the MMs of the two polymers. Other parameters such as the pore radius were fitted to the experimental values.

Figure 4a compares $M_{w}$ for eluted fractions calculated and observed experimentally in Exp-D. $M_{w}$ calculated in the simulation agrees well with the one observed in the experiment except for the portion of the relative cumulative mass close to 1 . This agreement indicates that the polymer was predominantly separated under the HOPC mechanism.

In Figure $4 \mathrm{~b}$, the pattern of change in $M_{w} / M_{n}$ with increasing cumulative mass is similar for calculation and experiment, but $M_{w} / M_{n}$ are quite different, possibly due to the assumption in our calculation that the original polymers before mixing have no MMD.

\section{Adsorption of PEG on CPG}

As Figure 4a shows, $M_{w}$ of late PEG fractions in Exp$D$ drastically increased as the relative cumulative mass approaches unity. The ratio of the peak height of PEG3K to that of PEG2K in the SEC chromatogram for these late fractions was greater than the ratio obtained for the injected PEG mixture. MMDs of the late fractions were close to the MMD of PEG3K. Similar behavior was found in Exp-C and Exp-E. The behavior was not observed in Exp-A in which less polar packing material $\mathrm{CPG}-\mathrm{C}_{8}$ was used. The same was observed in Exp-C, -D, and -E cannot be explained in the separation mechanism of HOPC.

According to the literature, silica surface adsorbs PEG molecules dissolved in water. ${ }^{19-22}$ However, there are conflicting reports regarding the adsorption of PEG onto silica from dioxane. ${ }^{19,23}$

When dissolved polymer has a bimodal MMD, high and low MM components compete for adsorption from solution. ${ }^{24,25}$ Although lower MM components are adsorbed onto surfaces first, many are eventually displaced by higher MM components. In equilibrium, higher MM components are adsorbed more preferentially than lower ones. In this investigation, the concentrations of PEGs 


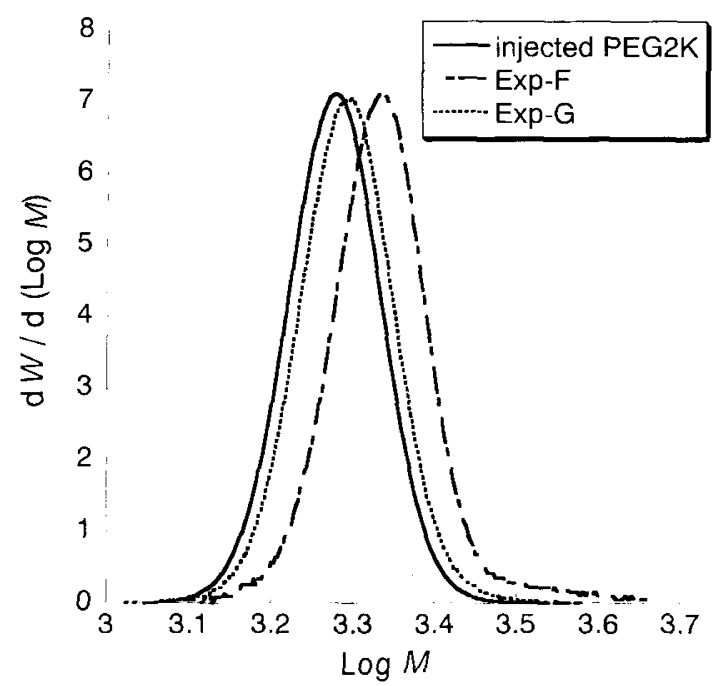

Figure 5. Comparison of molecular mass distribution between PEG2K injected (solid line), fraction 1 in Exp-F (dash-dotted line), and in Exp-G (dotted line).

were high, and therefore the equilibrium states would be attained quickly.

Two competitive partitioning processes should thus take place during the separation process : HOPC partitioning based on the osmotic pressure and size-exclusion, and partitioning based on adsorption. In separation with CPG-OH (Exp-C, -D, and -E), the following separation mechanism should apply: as the front end of the injected solution moved forward, some highest MM PEG components were adsorbed onto the CPG-OH surface. These components were attached to the pore surface when the solution in the mobile phase was concentrated to suppress desorption of the PEG components. The rest of PEG molecules were partitioned according to the HOPC separation. At the final stages of the separation, the mobile phase lowered the concentration to release the adsorbed PEG components to the mobile phase. Higher MM components enriched on the surface should have been retained onto the surface longer than lower ones. ${ }^{26}$ These components were collected as late fractions, giving rise to the anomalous behavior observed.

\section{Separation of Monodisperse PEG}

We fractionated each of PEG2K and PEG3K with an already narrow MMD in Exp-F through Exp-I. 1,4Dioxane as eluent and $\mathrm{CPG}_{\mathrm{C}} \mathrm{C}_{8}$-packed column were used in Exp-F and Exp-H. Water as eluent and CPG$\mathrm{OH}$-packed column were used in Exp-G and Exp-I. Figure 5 compares differential MMDs for fraction 1 (Exp-F and Exp-G) and injected PEG2K, all calculated from the SEC chromatograms. Fraction 1 of Exp-F had higher MM than that of Exp-G and the injected sample, but there was almost no change in the polydispersity index $M_{w} / M_{n}$. Fractionation of PEG3K (Exp-H and Exp-I) produced similar results in MMs and MMDs.

SEC chromatograms give $M_{w} / M_{n}$ larger than true values because of the band broadening inherent in the real liquid chromatography. To investigate differences in $M_{w}$ ' $M_{n}$ in more detail, we measured MALDI-TOFMS for the original PEGs and fractionated PEG samples.

Figure 6 shows the MALDI-TOFMS spectra of PEG2K
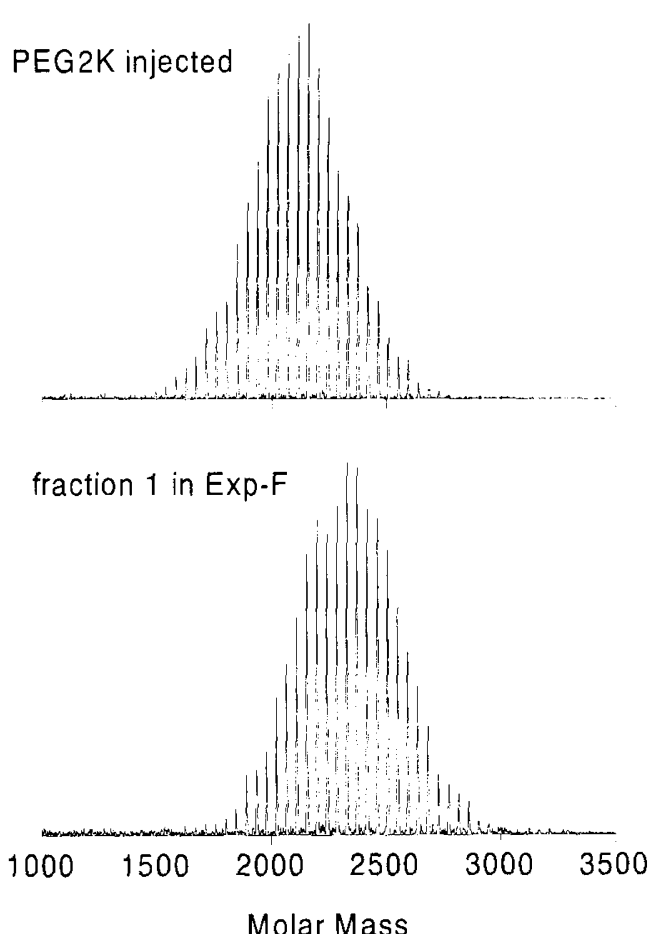

Figure 6. Comparison of MALDI-TOFMS spectra between PEG$2 \mathrm{~K}$ injected (upper) and fraction 1 in Exp-F (bottom).

injected (upper) and fraction 1 in Exp-F (bottom). MMs and MMDs calculated from these spectra were $M_{w}=$ $2100, M_{w} / M_{n}=1.010$ for PEG2K injected, and $M_{w}=2340$, $M_{w} / M_{n}=1.011$ for fraction 1 in Exp-F, respectively. Again, fraction 1 in Exp-F had higher MM compared with fraction 1 in Exp-G and the injected sample, but there was almost no difference in $M_{w} / M_{n}$. This indicates that the present HOPC method is not effective for preparing monodisperse polymer samples from already narrowly distributed samples. We need to improve the HOPC method or optimize separation conditions.

\section{CONCLUSION}

PEG samples with a bimodal MMD were fractionated by HOPC with 1,4-dioxane as eluent and $\mathrm{CPG}-\mathrm{C}_{8}$ as column packing material. To select a good combination of the eluent and packing material is essential. For PEG, less polar CPG surface and 1,4-dioxane are preferable to polar CPG surface and water because adsorption between PEG molecules and polar surface of CPG degrades the resolution. Finally, using HOPC, PEG fractions with higher MM and a narrow MMD could be fractionated from PEG with a narrow MMD. To obtain narrower MMD samples, we must improve the HOPC method.

\section{REFERENCES AND NOTES}

1. J. M. Barrales-Rienda, A. Bello, P. Bello, and G. M. Guzman, in "Polymer Handbook," 4th ed, J. Brandrup, E. H. Immergut, and E. A. Grulke, Ed., John Wiley, New York, N.Y., 1999, VII327.

2. I. Teraoka and M. Luo, Trends in Polym. Sci., 5, 258 (1997).

3. M. Luo and I. Teraoka, Polymer, 39, 891 (1998).

4. M. Luo and I. Teraoka, Macromolecules, 29, 4226 (1996).

5. A. Dube and I. Teraoka, Isolation and Purification, in press. 
6. Y. Xu, I. Teraoka, L. Senak, and C.-S. Wu, Polymer, 40, 7359 (1999).

7. J. des Cloizeaux and G. Jannink, "Polymers in Solution: Their Modelling and Structure", Clarendon Press, Oxford, 1990.

8. M. Daoud and P. G. de Gennes, J. Phys. (Paris), 38, 85 (1977).

9. I. Teraoka, Z. Zhou, K. H. Langley, and F. E. Karasz, Macromolecules, 26, 3223 (1993).

10. I. Teraoka, Z. Zhou, K. H. Langley, and F. E. Karasz, Macromolecules, 26, 6081 (1993).

11. S. Kawaguchi, G. Imai, J. Suzuki, A. Miyahara, T. Kitano, and K. Ito, Polymer, 38, 2885 (1997)

12. M. Kurata and Y. Tsunashima, in "Polymer Handbook," 4th ed, J. Brandrup, E. H. Immergut, and E. A. Grulke, Ed,, John Wiley, New York, N.Y., 1999, VII-1.

13. M. T. Bishop, K.H. Langley, and F. E. Karasz, Macromolecules, 22, 1220 (1989).

14. A. Dube and I. Teraoka, Macromolecules, 30, 7753 (1997).

15. S. Matsuyama, H. Nakahara, S. Kinugasa, and I. Teraoka, to be submitted.

16. D. R. Bloch, in "Polymer Handbook," 4th ed, J. Brandrup, E. H. Immergut, and E. A. Grulke, Ed., John Wiley, New York, N.Y., 1999, III-59.
17. T. Ohta and Y. Oono, Phy. Lett., 89A, 460 (1982).

18. From personal discussion between Teraoka and Wang, $R(X)=\left(1+0.27623 X+0.028645 X^{2}\right)^{(-1 / 16)}$

where $X$ is reduced concentration and $R(X)=R_{\mathrm{G}} / R_{\mathrm{G}, 0}$ at concentration $X$. Although this equation is valid only for a monodisperse system, we used it because the polydispersity in this experiment was small.

19. G. J. Howard and P. McConnell, J. Phys. Chem., 71, 2974 (1967).

20. M. Kawaguchi, H. Kawaguchi, and A. Takahashi, J. Colloid Interface Sci., 124, 57 (1988).

21. P. Trens and R. Denoyel, Langmuir, 9, 519 (1993).

22. J. C. Dijt, M. A. Cohen Stuart, and G. J. Fleer, Macromolecules, 25, 5416 (1992).

23. M. Kawaguchi, T. Hada, and A. Takahashi, Macromolecules, 22, 4045 (1989).

24. K. Furusawa, K. Yamashita, and K. Konno, J. Colloid Interface Sci., 86, 35 (1982).

25. M. Kawaguchi, S. Hattori, and A. Takahashi, Macromolecules, 20, 178 (1987)

26. H. Pasch and B. Trathnigg, "HPLC of Polymers", Springer, Berlin, 1997. 